\title{
Role of the synthesis route on the properties of hybrid LDH-graphene as basic catalysts
}

\author{
Mayra G. Álvarez ${ }^{1,2}$, Didier Tichit ${ }^{2}$, Francesc Medina ${ }^{1}$, Jordi Llorca ${ }^{3}$ \\ ${ }^{1}$ Department of Chemical Engineering, Universitat Rovira i Virgili, 43007 Tarragona, \\ Spain \\ ${ }^{2}$ Institut Charles Gerhardt, Equipe Materiaux Avances pour la Catalyse et la Sante, \\ 34296 Montpellier Cedex 5, France \\ 3 Institute of Energy Technologies and Centre for Research in Nanoengineering, \\ Technical University of Catalonia, 08028 Barcelona, Spain
}

\begin{abstract}
Layered double hydroxides (LDH or HT) or their derived mixed oxides present marked acid-base properties useful in catalysis, but they tend to agglomerate inducing a weak accessibility to the active sites. In this study we report the preparation and characterization of HT/Graphene (HT/rGO) nanocomposites as active and selective basic catalysts for the acetone condensation reaction. The graphene high specific surface area and structural compatibility with the HT allowed increasing the number and accessibility of the active sites and activity.

Two series of $\mathrm{HT} / \mathrm{rGO}$ nanocomposites with $0.5 \leq \mathrm{HT} / \mathrm{rGO} \leq 10$ mass ratio were prepared by: i) direct HT coprecipitation in the presence of GO; ii) self-assembly of preformed HT with GO. The prepared HT/rGO nanocomposites were dried either in air at $80{ }^{\circ} \mathrm{C}$ or freeze-dried.

A series of characterizations showed the great influence of the preparation method and $\mathrm{HT} / \mathrm{rGO}$ mass ratio on both the nanocomposite structure and catalytic activity. An optimum activity was observed for a $\mathrm{HT} / \mathrm{rGO}=10$ catalyst. Particularly, the highest catalytic activity was found in those nanocomposites obtained by coprecipitation and freeze dried ( 3 times more active than bulk HT) which can be connected to their structure with a better accessibility to the basic sites.
\end{abstract}




\section{Introduction}

Graphene has been widely studied because of its unique properties and provides huge possibilities for the modification or functionalization of its carbon backbone making possible the fabrication of graphene-based hybrid materials.

One attractive possibility for catalytic applications is its combination with layered double hydroxides (also known as hydrotalcites, HTs) leading to HTs-graphene hybrid nanocomposites. HTs have been indeed extensively investigated as catalysts for a wide sort of chemical reactions due to their large variety of composition ${ }^{1,2}$ which allows controlling their activity. HTs and the derived mixed oxides obtained by thermal decomposition are mainly known as basic catalysts with unique versatile properties although limited by the accessibility to the active sites due to their strong tendency of these materials to form large aggregates.

Several synthesis methods have been used to obtain poorly aggregated HTs particles of controlled size improving the accessibility to the acid-base pair active sites ${ }^{3,4}$. Recently, other approaches have been developed based on the dispersion of the HT phase on different supports leading to HT nanocomposites. ${ }^{5,6,7,8,9}$ The combination of (nano)carbon supports and HTs can lead to catalysts with improved properties such as high mechanical strength, surface area, thermal conductivity and chemical reactivity provided both by the carbon nanostructure and the HTs. For example, HT/CF nanocomposites obtained by growing MgAlHTs in situ on carbon fibers (CF) were efficient catalysts for the transesterification reaction of glycerol with diethyl carbonate into glycerol carbonate. ${ }^{10}$ HT crystals deposited inside a mesoporous carbon materials (CMK-41) were highly active catalysts in Claisen-Schmidt condensation. ${ }^{6}$ Winter et al. ${ }^{11}$ and Álvarez et al. ${ }^{12}$ investigated the catalytic activity of $\mathrm{MgAlHT} / \mathrm{CNF}$ nanocomposites obtained by direct coprecipitation of MgAlHTs on the surface of carbon nanofibers (CNFs). Their specific activity were more than four and hundred times higher in the acetone self-condensation ${ }^{12}$ and the glycerol transesterification reaction with diethyl carbonate ${ }^{13}$, respectively, than the unsupported MgAlHTs.

The common features of these hybrid materials are a small particle size and a good dispersion of the HT active phase, together with other factors such as tunable polarity.

In the last few years several studies dealing with the preparation of HT-graphene hybrid materials were focused on the applications as supercapacitors, electrocatalysts and photocatalysts but, to our knowledge, applications involving the acid-base properties in catalysis have not been reported so far. Hence, the development of HT-graphene materials with controlled catalytic properties for chemical synthesis is an interesting and promising field where intensive research is still to be carried out.

Herein, we present a study on the preparation of HT-reduced graphene oxide (HT/rGO) hybrids by in situ coprecipitation of Mg-Al HT on graphene oxide (GO) or by selfassembly of dispersed GO and pre-formed HTs in aqueous media. It must be pointed out the lamellar structure of both components materials (i.e. GO and HT) and their opposite 
surface charge which can lead to the formation of nanohybrids with enhanced HT phase dispersion and high mechanical and thermal stabilities able to improve the performances in base catalyzed reactions. We particularly studied the influence of the preparation method on the physico-chemical features of the HT/rGO hybrids and their catalytic activity. The effect of the HT content on the structural, textural and basic properties has been examined and fully characterized. The activity of the thermally treated HT/GO hybrids in the base-catalyzed self-condensation reaction of acetone has been investigated.

Although one work has revealed the basic features of this kind of materials to be used as $\mathrm{CO}_{2}$ adsorbents, ${ }^{13}$ here it is reported for the first time, to the authors' knowledge, the implementation of these properties in base-catalyzed reactions and the influence of the preparation method of the $\mathrm{HT} / \mathrm{rGO}$ hybrids.

\section{Experimental}

\subsection{Materials}

Natural flake graphite (carbon content: 99.8\%, -325 mesh) and $\mathrm{KMnO}_{4}(98 \%)$ were purchased from Alfa Aesar. $\mathrm{H}_{2} \mathrm{O}_{2}$ (30\%), $\mathrm{H}_{2} \mathrm{SO}_{4}(98 \%), \mathrm{NaNO}_{3}$ (analytical grade), $\mathrm{Mg}\left(\mathrm{NO}_{3}\right)_{2} \cdot 6 \mathrm{H}_{2} \mathrm{O}(99 \%), \mathrm{Al}\left(\mathrm{NO}_{3}\right)_{3} \cdot 9 \mathrm{H}_{2} \mathrm{O}(98.5 \%), \mathrm{NaOH}(>97 \%)$ and $\mathrm{HCl}(37 \%)$ were purchased from Sigma-Aldrich and used without further purification. Deionized water was used throughout the experiments.

\subsection{Synthesis of Graphene Oxide}

Graphene oxide (GO) is commonly used as an easily water-dispersible precursor to graphene $(\mathrm{G})$ or reduced graphene oxide (rGO). GO was synthesized using a modified Hummers method. ${ }^{14}$ Typically, $10 \mathrm{~g}$ of natural graphite and $5 \mathrm{~g}$ of $\mathrm{NaNO}_{3}$ were added to $230 \mathrm{~mL}$ of $\mathrm{H}_{2} \mathrm{SO}_{4}$. The temperature of the mixture was kept at $5{ }^{\circ} \mathrm{C}$ and then $30 \mathrm{~g}$ of $\mathrm{KMnO}_{4}$ was slowly added into the suspension. After $30 \mathrm{~min}$, the temperature was increased to $35{ }^{\circ} \mathrm{C}$ and the mixture was kept for another 30 min after which $460 \mathrm{~mL}$ of Milli-Q water was added. Then, the temperature was increased to $90^{\circ} \mathrm{C}$ for $1 \mathrm{~h}$. The resulted suspension was then diluted with addition of $700 \mathrm{~mL}$ of $\mathrm{H}_{2} \mathrm{O}$ and treated with 30 $\mathrm{mL}$ of a $30 \mathrm{wt} \% \mathrm{H}_{2} \mathrm{O}_{2}$ solution. The mixture was then cooled down to room temperature and repeatedly centrifuged and washed, first with a $5 \mathrm{wt} . \% \mathrm{HCl}$ solution and second with $\mathrm{H}_{2} \mathrm{O}$ until $\mathrm{pH} \approx 5$. The resulted solid was dried at $40^{\circ} \mathrm{C}$. The $\mathrm{GO}$ dispersion in water $(2.5$ $\mathrm{mg} / \mathrm{mL}$ ) was prepared by submitting the suspension to mechanical stirring for $1 \mathrm{~h}$ and subsequent ultrasonic treatment for $1 \mathrm{~h}$ to further exfoliate the GO. Stable brown colloidal suspension of GO layers was thus obtained.

\subsection{Synthesis of $\mathrm{MgAl} H \mathrm{HT}$}

$\mathrm{MgAl} \mathrm{HT}(\mathrm{Mg} / \mathrm{Al}=3)$ was synthesized by coprecipitation at constant $\mathrm{pH}$ as described elsewhere. ${ }^{15}$ Typically, $120 \mathrm{mmol}$ of $\mathrm{Mg}\left(\mathrm{NO}_{3}\right)_{2} \cdot 6 \mathrm{H}_{2} \mathrm{O}$ and $40 \mathrm{mmol}$ of $\mathrm{Al}\left(\mathrm{NO}_{3}\right)_{3} \cdot 9 \mathrm{H}_{2} \mathrm{O}$ were dissolved in $300 \mathrm{~mL}$ of distilled water and added dropwise into a glass vessel which initially contained $200 \mathrm{~mL}$ of deionized water. The $\mathrm{pH}$ was controlled by adding a $2 \mathrm{M}$ $\mathrm{NaOH}+0.5 \mathrm{M} \mathrm{Na}_{2} \mathrm{CO}_{3}$ aqueous solution and was kept at 10. Both solutions were mixed 
under vigorous stirring and the white suspension was stirred overnight at either r.t or 180 ${ }^{\circ} \mathrm{C}$. The precipitated solid was filtered and washed several times with water $(\approx 3 \mathrm{~L})$ until neutral $\mathrm{pH}$ and dried at $80{ }^{\circ} \mathrm{C}$ during $24 \mathrm{~h}$. These samples were hereafter noted as and HT180.

\subsection{Synthesis of HT/rGO hybrids by direct coprecipitation}

The corresponding amounts of salts $\left(\mathrm{Mg}\left(\mathrm{NO}_{3}\right)_{2} \cdot 6 \mathrm{H}_{2} \mathrm{O}\right.$ and $\left.\mathrm{Al}\left(\mathrm{NO}_{3}\right)_{3} \cdot 9 \mathrm{H}_{2} \mathrm{O}\right)$ were added to $100 \mathrm{~mL}$ of $\mathrm{GO}$ dispersion in water $(2.5 \mathrm{mg} / \mathrm{mL})$ to obtain $\mathrm{HT} / \mathrm{rGO}$ hybrid materials with a HT:GO mass ratio of 0.5:1 and 10:1. Subsequently, a $2 \mathrm{M} \mathrm{NaOH}+0.5 \mathrm{M} \mathrm{Na}_{2} \mathrm{CO}_{3}$ aqueous solution was added to the mixture to fix the $\mathrm{pH}$ at 10 under continuous stirring. The resulting dark-brown suspension was hydrothermally treated in autoclave at $180{ }^{\circ} \mathrm{C}$ under autogenous pressure for $12 \mathrm{~h}$. The hydrothermal treatment performed in basic media resulted in the GO reduction whilst forming the HT and the solution turned from brown to black. The suspension was centrifuged, washed several times with $\mathrm{H}_{2} \mathrm{O}$. The samples were dried by either freeze drying or in static air at $80^{\circ} \mathrm{C}$. They were noted HT/rGO-xCFD and HT/rGO-xC80 (with $\mathrm{x}=0.5$ or 10; $\mathrm{C}$ : coprecipitation; FD: freeze drying and 80: drying at $80{ }^{\circ} \mathrm{C}$ ).

\subsection{Synthesis of HT/rGO hybrids by self-assembly}

Required amount of a previously synthesized $\mathrm{MgAl}-\mathrm{HT}(\mathrm{Mg} / \mathrm{Al}=3)$ was dispersed in water and added to the $\mathrm{GO}$ dispersion $\left(2.5 \mathrm{mg}\right.$ per $\mathrm{mL}$ of $\left.\mathrm{H}_{2} \mathrm{O}\right)$ so that hybrid materials with a HT:GO mass ratio of 0.5:1 and 10:1 were obtained. The suspension was submitted to ultrasound for $15 \mathrm{~min}$ and then hydrothermally treated in autoclave at $180{ }^{\circ} \mathrm{C}$ under autogenous pressure for $12 \mathrm{~h}$. The hybrid materials were separated from the aqueous media and dried either in static air at $80{ }^{\circ} \mathrm{C}$ or freeze dried. They were noted HT/rGOxSAFD and HT/rGO-xSA80 (with SA: self-assembly).

\subsection{Characterization techniques}

PXRD measurements were made in a Bruker-AXS D8-Discover diffractometer with a vertical theta-theta goniometer and a Vantec linear detector. Monochromatic $\mathrm{Cu} \mathrm{K \alpha}$ radiation was obtained from a copper X-ray tube operated at $40 \mathrm{kV}$ and $40 \mathrm{~mA}$ and a $\mathrm{Ni}$ monochromator. Data were recorded over a $2 \theta$ range of $5-70^{\circ}$ with an angular step of $0.02^{\circ}$ at $10.6 \mathrm{~s} / \mathrm{step}$.

Specific surface areas were determined by $\mathrm{N}_{2}$ adsorption at $-196^{\circ} \mathrm{C}$ using a Micromeritics ASAP 2020 automatic analyzer. Samples were previously degassed in situ at $150{ }^{\circ} \mathrm{C}$ under vacuum for $15 \mathrm{~h}$. Surface areas were calculated using the Brunauer-Emmet-Teller (BET) method over a p/p0 range where a linear relationship was maintained.

Morphology of the samples was analyzed by electron microscopy. Transmission electron microscopy (TEM) was performed in the bright field using a JEOL JEM-2100 instrument at an accelerating voltage of $100 \mathrm{kV}$. The samples were prepared by dispersing the asprepared catalysts in ethanol and then drop casting the suspension on a standard $3 \mathrm{~mm}$ holey copper grid and letting the ethanol evaporate at room temperature. Those samples 
with the highest rGO content were also analyzed with scanning transmission electron microscopy (STEM) operated under high angle annular dark-field mode (HAADF) on a Tecnai $\mathrm{G}^{2}$ F20 S-TWIN microscope equipped with a field emission electron source. It was operated at $200 \mathrm{kV}$. Samples were deposited from alcohol suspensions onto holeycarbon $\mathrm{Cu}$ grids. The point-to-point resolution was $0.24 \mathrm{~nm}$ and the resolution between lines was $0.10 \mathrm{~nm}$. High Resolution Transmission Electron Microscopy (HRTEM) was performed at an accelerating voltage of $200 \mathrm{kV}$ in a JEOL $2010 \mathrm{~F}$ instrument equipped with a field emission source. The point-to-point resolution was $0.19 \mathrm{~nm}$, and the resolution between lines was $0.14 \mathrm{~nm}$. Samples were dispersed in alcohol in an ultrasonic bath, and a drop of supernatant suspension was poured onto a holey carbon-coated grid. Images were not filtered or treated by means of digital processing, and they correspond to raw data.

The actual HT:rGO ratio was measured by thermogravimetric analysis (TGA) using a Perking Elmer Pyris STA 6000. Typically, $5 \mathrm{mg}$ samples were in situ dried at $80^{\circ} \mathrm{C}$ for $20 \mathrm{~min}$ and then heated from 80 to $900{ }^{\circ} \mathrm{C}$ at $1{ }^{\circ} \mathrm{C} \mathrm{min}{ }^{-1}$ in $20 \mathrm{~mL} \mathrm{~min}{ }^{-1}$ of air.

The basicity measurements (volumetric $\mathrm{CO}_{2}$ adsorption) were performed with a Micromeritics ASAP $2010 \mathrm{C}$ apparatus in the range $0-100 \mathrm{mmHg}$ at $30^{\circ} \mathrm{C}$. Samples were previously pretreated in situ at $450{ }^{\circ} \mathrm{C}$ in vacuum for $12 \mathrm{~h}$. Afterwards, $\mathrm{CO}_{2}$ was dosed with small increments. The total number of accessible basic sites was determined by taking the amount of $\mathrm{CO}_{2}$ chemisorbed at zero pressure $\left(\mathrm{cm}^{3} / \mathrm{g}\right.$ STP) obtained by extrapolation of the linear part of the uptake isotherm.

\subsection{Catalytic activity tests}

The catalytic activity of $\mathrm{HT} / \mathrm{rGO}$ hybrids was evaluated by aldol condensation of acetone as a probe reaction. Typically, the reaction was performed in a $4 \mathrm{~mL}$ flask at $0{ }^{\circ} \mathrm{C}$ with the temperature maintained by means of an ice bath. The $27.5 \mathrm{mmol}$ of acetone and 137 $\mu \mathrm{L}$ of isooctane (used as internal standard) were placed into the flask, and freshly activated $\mathrm{HT} / \mathrm{rGO}$ hybrid samples ( $1.5 \mathrm{wt} \%$ respect to acetone) were added in the acetone. The operations above were carried out under $\mathrm{N}_{2}$ atmosphere in order to exclude atmospheric carbon dioxide. The mixture of products was analyzed by a gas chromatograph (GC QP2010, Shimadzu) and products were identified by GC-mass spectrometer (GC-MS QP2010, Shimadzu) and further confirmed by ${ }^{1} \mathrm{H}$ NMR.

\section{Results and discussion}

PXRD patterns of graphite, graphene oxide (GO) and reduced graphene oxide (rGO) (Figure S1) confirmed the oxidation of graphite to GO evidenced by the decrease of the interlayer distance due to the loss of the oxidative debris produced during oxidation process and the reduction of GO into $\mathrm{rGO}$ after hydrothermal treatment. The rGO pattern presented a broad peak between $2 \theta=20-30^{\circ}$ indexed as (002) plane corresponding to a disordered restacking of exfoliated rGO sheets. 
PXRD patterns of all the prepared HT/rGO hybrids are displayed in Figure 1 . Their shape greatly depends on the HT:rGO mass ratio. In all cases the typical reflections corresponding to the 2D HT material are clearly observed. It is the only phase detected at higher HT content (HT:rGO = 10:1) with particularly intense (003) and (006) reflections below $30^{\circ} 2 \theta$. These peaks are less intense with the presence of an additional broad peak between $2 \theta=20-30^{\circ}$ corresponding to rGO in those samples with lower HT content (HT:rGO $=0.5: 1$ ). Notably it is more visible in the case of the self-assembly prepared hybrids.

Most striking is, in these latter samples, the shift toward lower $2 \theta$ value of the reflection assigned to the HT (003) plane with respect to the as-synthesized HT, accounting for an increase of the basal spacing from $7.72 \AA$ to $8.30 \AA$ (Figure $1 \mathrm{~A}$ and Table 1). If crystallographic layer thicknesses of HT and rGO are taken into account ( $4.8 \AA$ and 3.5 $\AA$, respectively) this increase of the basal spacing is consistent with an exchange of the initial carbonate anions by rGO sheets with a face-to-face orientation of the HT and rGO layers to form a sandwich-type structure. These self-assembly prepared hybrids also present additional weak harmonics, as well as (100), (110) and (113) peaks showing that the structure of the LDH nanosheets is maintained as recently reported in a HT/rGO hybrid. ${ }^{16,17}$. Besides, the small harmonics found in these samples at lower $2 \theta$ angles (marked by arrows in Figure 1A) reinforce the hypothesis of formation of a sandwichtype superstructure. The basal spacing of this superstructure may be affected by the degree of functionalization of rGO layers, the electrostatic interaction between $\mathrm{rGO}$ and HT layers as well as the water content into the hybrid layers.

On the other hand, no differences were observed in the PXRD of the samples with higher HT content, i.e. with HT:rGO = 10:1. In this case, all the synthesized hybrids presented the typical diffraction planes of the HT with a basal peak at ca. $7.72 \AA$, consistent with the presence of carbonate anions provided by the alkaline synthesis solution into the interlayer space.

As could be expected, intensity of HT peaks increases with the HT content in the hybrid materials. In addition, the width of the HT peaks increases in the hybrids in comparison with bare HT, and increases with the rGO content in the hybrid. Further, the highest width of HT peaks have been found in the samples prepared by co-precipitation and freeze dried, the samples prepared by self-assembly being more crystalline, showing a noticeable effect of the preparation route on the crystallinity of the HT/rGO samples.

These results demonstrate a clear influence not only of the preparation and drying methods on the structural features of $\mathrm{HT} / \mathrm{rGO}$ hybrids, but also of the relative content of the GO and HT phases.

To further investigate on the morphology and microstructure of the HT/rGO hybrids, SEM and TEM analysis were performed. Selected SEM images are presented in Figure 2 showing that the hybrids with a low HT content $(\mathrm{HT}: \mathrm{rGO}=0.5: 1)$ revealed a stacked structure where HT and rGO phases cannot be easily distinguished. This was found especially in sample $\mathrm{HT} / \mathrm{rGO}-0.5 \mathrm{C} 80$, i.e. prepared by coprecipitation and dried at $80{ }^{\circ} \mathrm{C}$ 
where the structure consists of highly packed HT and rGO sheets. These images suggest the formation of a poorly porous material. On the other hand, sample HT/rGO-0.5SA80 (prepared by self-assembly and dried at $80^{\circ} \mathrm{C}$ ) presented a house-of-cards-type stacked morphology where a number of hexagonal platelet-like crystallites typical of HT material could be seen, which strongly suggests the formation of a mesoporous structure. These findings are consistent with the specific surface areas found from $\mathrm{N}_{2}$ physisorption measurements (Table 1). Although the HT materials are more electron-dense than graphene, the direct interpretation by TEM based on contrast was not possible in this series of samples since both the building materials rGO and HT exhibit similar features depending on the thickness of the rGO layers and their bending. For that reason, STEMHAADF was carried out on samples with high rGO content, in addition to TEM, to get a better understanding on the microstructure of the composite materials.

STEM-HAADF and TEM micrographies revealed different structures depending both on the synthesis and drying protocols followed. For instance, sample HT/rGO-0.5C80 is mainly constituted by rGO sheets and HT aggregates in contact. Figure $3 \mathrm{~A}$ shows a general TEM view of the sample where rGO and HT domains have been identified and a higher magnification image is provided in Figure 3B. However, the occurrence of separate domains of rGO and HT has also been found as showed in STEM-HAADF image (Figure 3C), revealing certain heterogeneity of the hybrid material. Other TEM images are provided in Figures S2A and B in the supplementary information, which show the occurrence of domains presenting an intimate contact between rGO and HT but also the existence of completely separate domains. In contrast, the sample prepared by coprecipitation and freeze dried (HT/rGO-0.5CFD) presents a very homogeneous structure with highly dispersed HT nanoplatelets on the rGO surface (Figure 3D-F). Measurements of the HT nanoplatelets revealed a mean crystal domain in the range of $40-60 \mathrm{~nm}$ in the $\mathrm{xy}$ direction and of about $8-10 \mathrm{~nm}$ in the $\mathrm{z}$ direction. HT nanoplatelets in this sample are absolutely in contact with the rGO sheets as seen in the higher magnification TEM images showing that most of the HT platelets are oriented face-on (i.e., with xy plane parallel to the rGO substrate). This can be perfectly seen in the TEM images showed in Figures 3D and E and in the STEM-HAADF image (Figure 3F).

Regarding to the self-assembly prepared samples (HT/rGO-0.5SA80 and HT/rGO$0.5 \mathrm{SAFD}$ ), both presented a heterogeneous structure independently of the drying method, exhibiting domains where intimate contact between the HT platelets and the rGO layers, but also separate domains are produced. Figure 4A shows an example of a domain in sample HT/rGO-0.5SA80 where contact between HT and rGO is manifested and a higher magnification view is provided in figure $4 \mathrm{~B}$. Figures $4 \mathrm{C}$ and $4 \mathrm{D}$ show the STEM-HAADF confirming a good contact between HT and rGO according to a good affinity between the both building components and suggesting the formation of the hybrid HT/rGO structure. In addition, the HT platelets found in these domains presented crystal sizes of about 40$60 \times 10 \mathrm{~nm}$, similarly to those found in the previous sample (prepared by coprecipitation and freeze dried). Nonetheless, separate domains were also found in this HT/rGO0.5SA80 sample (Figure S3). In this domains, HT platelets presented crystal sizes of 
about 100-150 x 14-18 nm. Sample HT/rGO-0.5SAFD (prepared by self-assembly and freeze dried) presented similar features. TEM and STEM-HAADF of sample HT/rGO0.5SAFD can be found in supplementary information (Figure S4). However, no direct evidences of intercalation between HT and rGO sheets able to confirm the previous XRD observations have been found by this technique.

HRTEM images of the hybrid samples with the highest HT content (i.e., HT:rGO = 10:1) are shown in Figure 5 and revealed the differences existing among hybrids prepared by the different routes. HT/rGO-10 hybrids prepared by coprecipitation presented a great homogeneity (Figure 5A), higher than those prepared by self-assembly (Figure 5B). In addition, the size of the HT platelets is notably smaller in the hybrids prepared by coprecipitation, where nanoplatelets in the range of 30-40 $\mathrm{nm}$ in the xy direction and about 4-6 nm in the $\mathrm{z}$ direction were observed, whereas most of the HT platelets in the hybrids prepared by self-assembly (identified by having a higher electron contrast) presented particles sizes higher than 100-200 $\mathrm{nm}$ in the xy direction. As an example, compared detailed structural information about the growth between HT and rGO in HT/rGO-10CFD and HT/rGO-10SAFD nanocomposites is provided in Figures 5C and D, which shows lattice-fringe images. Two types of lattice fringes are recognized (inset in Figures 5C and D): one at $3.5 \AA$ corresponding to the (002) planes of graphene and a set of planes at 2.28 and $1.95 \AA$, which corresponds to the HT structure. The HT crystals appeared intimately assembled into the graphene (particularly in samples prepared by coprecipitation); however, no apparent structural intercalation between both layered materials was observed. Further, alignment along the (001) crystallographic direction between HT particles and rGO was not found.

The specific surface areas of the as-prepared rGO/HT hybrid materials vary largely between 13 and $198 \mathrm{~m}^{2} \mathrm{~g}^{-1}$ (Table 1). It is noticeable that for a same preparation method (coprecipitation or self-assembly) the surface area is generally higher for the freeze dried samples than for those dried at $80{ }^{\circ} \mathrm{C}$. On the other hand, for a same preparation method and drying process, the surface area decreases at higher HT content, except in the case of the coprecipitated hybrids dried at $80^{\circ} \mathrm{C}$. This latter feature is consistent with the HRTEM image showing highly packed HT and rGO sheets in the structure of HT/rGO-0.5C80 exhibiting a surface area of $13 \mathrm{~m}^{2} \mathrm{~g}^{-1}$, while the structure appears homogeneous with HT crystals in the range of 30-40 $\mathrm{nm} \times$ 4-6 nm intimately assembled into the graphene in the case of HT/rGO-10C80 exhibiting a surface area of $102 \mathrm{~m}^{2} \mathrm{~g}^{-1}$. Based also on the HRTEM characterization, it can be pointed out that the highest surface areas in the range $130-$ $200 \mathrm{~m}^{2} \mathrm{~g}^{-1}$ were reached in those samples showing heterogeneous structures exhibiting domains with intimate contact between the HT platelets and the rGO layers and also separate domains of large HT particles with sizes higher than $100 \mathrm{~nm}$ in the xy direction and $14 \mathrm{~nm}$ in the $\mathrm{z}$ direction (HT/rGO-0.5SA80, HT/rGO-0.5SAFD and HT/rGO10SAFD).

A nearly twofold increase of the specific surface areas reaching values in the range 170 $230 \mathrm{~m}^{2} \mathrm{~g}^{-1}$ occurs in the $\mathrm{rGO} / \mathrm{HT}$ materials after calcination of the hybrids. This is in agreement with the structural transformation of the $\mathrm{Mg}$ - $\mathrm{Al} \mathrm{HT}$ component into $\mathrm{MgAlO}$ 
mixed oxide of higher surface area. This is confirmed by the fourfold increase of surface area observed in the case of HT180 sample after calcination (Table 1).

The ATR-FTIR spectra (Fig. 6A) of parent GO revealed peaks at 865 and $1038 \mathrm{~cm}^{-1}$ respectively attributed to the asymmetric bending and stretching of $\mathrm{C}-\mathrm{O}-\mathrm{C}$ vibrations, of the epoxy group. ${ }^{18,19}$ The peak observed at $\sim 1730 \mathrm{~cm}^{-1}$ is due to the $\mathrm{C}=\mathrm{O}$ stretching from carboxylic acid and carbonyl moieties. ${ }^{19}$ The peaks at $\sim 3350 \mathrm{~cm}^{-1}$ (broad, with a shoulder at $\sim 3165 \mathrm{~cm}^{-1}$ ) and $1624 \mathrm{~cm}^{-1}$ can be assigned to O-H stretching and $\mathrm{H}-\mathrm{O}-\mathrm{H}$ bending of adsorbed water molecules, respectively. At a first glance, once the HT is present in the composite, the bands relative to the oxidized surface of GO disappeared (confirming GO reduction by hydrothermal treatment) and those corresponding to HT rose with its increasing amount. The spectra of the HT/rGO-10 family of samples indeed correspond to that of HT. This is in agreement with the behavior previously observed by XRD (Figure 1). Interestingly, the bands in the hydroxyl-stretching region between 2500 and $4000 \mathrm{~cm}^{-1}$ presented a shift in the hybrid nanocomposites compared to mere HT (Figure 6B). In fact, the band component analysis of the hydroxyl-stretching region corresponding to hybrids with higher amount of HT (HT/rGO-10 samples) revealed four different stretching modes, as early observed by Frost et al. ${ }^{20}$ (Figure S5): a small contribution at ca. $3600 \mathrm{~cm}^{-1}$ assigned to free $\mathrm{OH}$ groups; an intense band around 3500 $\mathrm{cm}^{-1}$ due $\mathrm{M}-\mathrm{OH}$ stretching modes (mainly due to the $\mathrm{Al}-\mathrm{OH}$ bond with a small influence of nearby $\mathrm{Mg}$ cations) and two bands around 3300 and $3000 \mathrm{~cm}^{-1}$ interpreted as hydrogenbonded interlayer water and interlayer carbonate-water bridging mode, respectively. Even though all these contributions presented diverse shifts among the different samples, the stretching assigned to $\mathrm{M}-\mathrm{OH}$ contribution band showed notable band shifts depending on the drying method, revealing important structural information. Thus, the corresponding $\mathrm{M}-\mathrm{OH}$ contribution band in pure HT was found at $3453 \mathrm{~cm}^{-1}$, similarly to HT/rGO-10 composites dried in static air at $80^{\circ} \mathrm{C}$ (only a slight red-shift of ca. $5 \mathrm{~cm}^{-1}$ was observed). On the contrary, an important $\mathrm{M}-\mathrm{OH}$ blue-shift (around $20 \mathrm{~cm}^{-1}$ ) was observed for the HT/rGO-10 composites submitted to freeze drying. These results suggest the formation of more ordered structures by hydrogen bonding in samples dried in air, whereas freeze drying seems to affect ordering between HT and GO sheets avoiding hydrogen bond interaction and, hence, producing a M-OH blue-shift. In other words, freeze drying avoid both HT and rGO layers restacking as has been previously described in TEM images. These features were further reflected in their macroscopic aspects, so that the freeze-dried hybrids presented a low-dense fluffy conformation, meanwhile the hybrids dried in air at $80{ }^{\circ} \mathrm{C}$ showed a more rigid structure.

Deconvolution of hydroxyl-stretching band was not possible in the case of nanocomposites with low HT content due to the low intensity and wide wavenumber range of this band. However, a marked red-shift in the hybrids compared to bare HT could be observed indicating a strong interaction of the both components (i.e. HT and rGO) independently of synthesis or drying method. Nonetheless, this interaction seems to be higher in the case of hybrids dried at $80{ }^{\circ} \mathrm{C}$ than freeze dried, following the same trend than above described for the hybrids with high HT content. 
Raman spectra $(\lambda=514 \mathrm{~nm})$ of HT/rGO hybrids display two peaks, the $\mathrm{G}$ peak around $1590 \mathrm{~cm}^{-1}$ and the $\mathrm{D}$ peak around $1350 \mathrm{~cm}^{-1}$, typically assigned to the first-order scattering of the $\mathrm{E}_{2 \mathrm{~g}}$ mode observed for $\mathrm{sp}^{2}$ carbon domains, and amorphization and structural defects, respectively. ${ }^{21,22}$ Thus, the intensity ratio $I_{D} / I_{G}$ for bands $D$ and $G$ is a measure widely used to quantify disorder and defect quantity in graphitic materials. The $\mathrm{I}_{\mathrm{D}} / \mathrm{I}_{\mathrm{G}}$ ratio for the prepared hybrids (around 0.99 and 0.90 for samples with higher and lower HT contain, respectively) displayed an enhanced value compared to reduced GO (0.84), which indicates the presence of $\mathrm{sp}^{3}$ defects within the $\mathrm{sp}^{2}$ carbon network. Thus, it is plausible that HT presence provokes a randomly arrangement of the graphene sheets or hinders graphene re-stacking. In addition, FWHM in both peaks (D and G) broaden due to disorder and, further, bands intensity decreased indicating the presence of less crystalline domains (lower number of stacked rGO sheets), especially in those hybrids submitted to freeze drying. Red-shifts of $D$ and $G$ bands were also observed in the prepared hybrids compared to reduced GO indicating strong interactions between the components. ${ }^{23,24,25}$

\subsection{Acetone self-condensation}

The self-condensation of acetone was chosen as a model reaction to study the catalytic performance of the $\mathrm{HT} / \mathrm{rGO}$ hybrid. Moreover, it allows an interesting comparison with HT-CNF or HT-MWCNT nanocomposites already studied in the same reaction. ${ }^{10,12}$ The $\mathrm{HT} / \mathrm{rGO}$ hybrid materials were previously activated by calcination at $450{ }^{\circ} \mathrm{C}$ for $5 \mathrm{~h}$ then immediately placed into the reactor without exposure to ambient atmosphere in order to avoid $\mathrm{CO}_{2}$ contamination. Diacetyl alcohol (DAA) was the only reaction product obtained in all cases and no by-products, particularly mesityl oxide corresponding to DAA dehydration, or resulting from multiple condensation and cyclization such as phorone and isophorone were observed nor by GC-MS neither by ${ }^{1} \mathrm{H}-\mathrm{NMR}$.

$\mathrm{HT} / \mathrm{rGO}-\mathrm{xC} 80$ nanocomposites with increasing HT content, i.e. with HT:rGO $=0.5: 1,1: 1$, 5:1, 10:1 and 20:1 (see details in SI, Table S1 and Figure S1B), have been first tested. Results showed that catalytic activity was enhanced by increasing the HT content with a maximum found for $\mathrm{HT}: \mathrm{rGO}=10: 1$. This accounts for a regular increase in the amount of catalytically active basic sites until HT:rGO= 10:1 and then for a decrease of accessibility to the active sites at higher HT content probably due to aggregation of the HT particles. It can be underlined that the catalytic activity dramatically increases from HT:rGO $=0.5: 1$ to $1: 1$ the catalyst with the lowest HT content being poorly active (Figure 7A). During the reaction time of $480 \mathrm{~min}$, in any case, the equilibrium conversion of $23.1 \%$ was reached ${ }^{26}$; however, the catalytic performance showed by the calcined HT/rGO-xC80 catalysts with HT:rGO=5:1, 10:1 and 20:1 was superior to that of the calcined bare HT (HTc) or other HT-derived mixed oxides reported previously ${ }^{12,18,27,28}$. Bare GO and rGO did not show any catalytic activity under the reaction conditions tested, which confirms that HT is the active phase of the reaction.

These results point out the influence of the $\mathrm{HT} / \mathrm{rGO}$ mass ratio on the catalytic activity of the hybrid materials prepared by coprecipitation and drying at $80{ }^{\circ} \mathrm{C}$. However, as we 
have already shown that the preparation route and the drying method have a strong influence on the structure and morphology we also examine their influence on the catalytic performances focusing on the hybrids with HT:rGO $=0.5: 1$ and 10:1 which exhibit the lowest and the highest catalytic activity, respectively, when obtained by coprecipitation and drying at $80{ }^{\circ} \mathrm{C}$. The catalytic results are displayed in Figure $7 \mathrm{~B}$ and $\mathrm{C}$ and Table 2.

As expected, the different activated HT/rGO hybrids with mass ratio HT:rGO $=0.5: 1$, due to their low HT content and then the low number of basic sites, are poorly active in the self-condensation of acetone, with conversion values below $1 \%$, lower than that achieved with the mixed oxide obtained by activation of $\mathrm{HT}$ at $450{ }^{\circ} \mathrm{C}$. Moreover, in these hybrids, the large excess of rGO may hinder the accessibility to the basic sites. Differences have been found depending on the preparation route. The two coprecipitated samples; i.e. HT/rGO-0.5CFD and HT/rGO-0.5C80, freeze dried and dried at $80{ }^{\circ} \mathrm{C}$, respectively, indeed presented the greatest and the lowest catalytic activity. Though the samples have similar HT content, the higher specific area and the morphology of the freeze dried sample can account for a higher accessibility to the active sites. For HT/rGO$0.5 \mathrm{C} 80$, the SEM images as well as the low porosity suggest highly packed HT and rGO sheets with HT nanocrystals encapsulated between several layers of graphene (Figure 2A and $\mathrm{B}$ ) being thus inaccessible for the acetone molecules and preventing reactivity. In the case of HT/rGO-0.5CFD, the HT dispersion is enhanced (Figure 3D-F) and the acetone conversion greatly improved compared with $\mathrm{HT} / \mathrm{rGO}-0.5 \mathrm{C} 80$. The catalytic activity of the catalysts prepared by self-assembly is almost similar, whatever the drying method, in agreement with their close surface areas and morphologies.

The HT/rGO-0.5 nanocomposites are less active than the MgAlO mixed oxide (HTc) in contrast to the greatest activity found for HT-CNF or HT-MWCNT composites containing similar or even lower HT loadings ${ }^{10-12}$.This fact suggests a different coupling mode between the HT and rGO components than between the components of the HTCNF or HT-MWCNT composites.

More generally the low specific activity showed by the HT/rGO-0.5 catalysts besides the low number of basic sites may be attributed to several other factors. On the one hand, PXRD analysis of these samples suggests at least a partial layer-by-layer hybridization where the positively charged HT layers can bind with the negatively charged GO sheets, highly favored by the geometric compatibility between both 2D structures. This hypothesis is in good agreement with the results observed by FTIR. In such scenario, $\mathrm{MgAl}$ HT layers may be entirely occluded between two rGO layers and, hence, their active sites are almost inaccessible to the reaction medium. On the other hand, one may also consider a partial decomposition of rGO during activation, with carbon poisoning a part of the HT active centers. However, this later hypothesis could be discarded since no degasification from rGO has been observed at the activation conditions.

When the HT loading is increased to HT:rGO $=10: 1$, the catalytic performance was greatly improved and the acetone conversion was higher than that of the $\mathrm{MgAlO}$ mixed 
oxide (HTc) and of other HT-based catalysts reported in the literature. ${ }^{18-29}$ A slighter influence of the drying and synthesis methods than at low HT loading on the hybrids' catalytic performance was observed, the catalysts submitted to freeze drying presenting the higher DAA yields. Of particular interest is the case of the hybrid catalyst prepared by coprecipitation and freeze dried (HT/rGO-10CFD) which showed the best performance with an average DAA amount of $102.7 \mathrm{mmol} / \mathrm{g}_{\mathrm{HTc}}$; that is a specific activity (based on the weight of HT present in the hybrid catalyst) about 2.5 times higher than that of HTc. This result is consistent with observations done by FTIR and Raman spectroscopy, showing that samples submitted to freeze drying presented a different ordering between HT and GO sheets than samples dried at $80{ }^{\circ} \mathrm{C}$ resulting in less stacked nanostructures, which ensures the accessibility to the HT active sites during the reaction. This was already observed in case of hybrid catalyst with lower HT:rGO ratio (0.5:1) and freeze dried. In addition, the improved activity in catalyst HT/rGO-10CFD may also be due to the highly dispersed nanosized HT platelets as observed in XRD and HRTEM analysis. Such observations would be consistent with the hypothesis that the number of active sites is determined by the lateral size of the HT platelets ${ }^{5-9,11,12}$.

Because $\mathrm{CO}_{2}$ has been successfully applied as probe molecule to gain information about the number of basic sites in activated HTs, volumetric $\mathrm{CO}_{2}$ measurements at low pressures have been performed on the activated HT/rGO nanocomposites and the bulk HT catalysts (Table 2).

The $\mathrm{CO}_{2}$ adsorption capacity on the activated $\mathrm{HT} / \mathrm{rGO}$ nanocomposites significantly increased with the LDH content, in good agreement with the catalytic results. The amount of $\mathrm{CO}_{2}$ adsorbed on the $\mathrm{HT} / \mathrm{rGO}-0.5$ catalysts ranges from 63 to $157 \mu \mathrm{mol} \mathrm{CO}_{2} \cdot \mathrm{g}_{\text {ads. }}^{-1}$. Whereas, $\mathrm{CO}_{2}$ adsorbed on the HT/rGO-10 catalysts ranges from 361 to $407 \mu \mathrm{mol} \mathrm{CO} \mathrm{CO}_{2}$. $\mathrm{g}_{\text {ads. }}^{-1}$, a factor 2 times higher than that found for the bulk HTc, $206 \mu \mathrm{mol} \mathrm{CO} 2 \cdot \mathrm{g}_{\text {ads. }}^{-1}$. This is in accordance with earlier studies of HT/rGO nanocomposites ${ }^{13,30}$. In the analysis conditions, the $\mathrm{CO}_{2}$ adsorption capacity of the bare $\mathrm{rGO}$ was found to be negligible.

Significantly, $\mathrm{CO}_{2}$ adsorption of sample $\mathrm{HT} / \mathrm{rGO}-0.5 \mathrm{C} 80$ was about half that reached by the other samples in this series. This is consistent with the morphology and texture observed in this material. The similar $\mathrm{CO}_{2}$ adsorption of the rest of catalysts is in agreement with their close surface areas and morphology and can be ascribed to a higher dispersion of HT nanoplatelets. It is noticeable that their $\mathrm{CO}_{2}$ adsorption capacity is in the same range $\left(116-157 \mu \mathrm{mol} \mathrm{CO} 2 \cdot \mathrm{g}_{a d s .}^{-1}\right)$ than that of $\mathrm{HT}_{\mathrm{C}}\left(206 \mu \mathrm{mol} \mathrm{CO} \mathrm{CO}_{2} \cdot \mathrm{g}_{a d s .}^{-1}\right)$. However, when it is normalized per mass of HT $\left(350-475 \mu \mathrm{mol} \mathrm{CO}_{2} / \mathrm{g}_{\mathrm{LDH}}\right)$ it is significantly higher than HTc and HTs supported on $\mathrm{CF}^{10}, \mathrm{CNF}^{11,12}$ or MWCNT ${ }^{13}$ under the same operating conditions (c.a. 210-400 $\mu \mathrm{mol} \mathrm{CO}_{2} / \mathrm{g}_{\mathrm{LDH}}$ ), though being less catalytically active. This emphasize on different behavior of HT/rGO materials than other carbon-based materials such as HT-CNF or HT-MWCNT composites.

The amount of $\mathrm{CO}_{2}$ adsorbed on HT/rGO-10 nanocomposites, is 2-3 times higher than on HT/rGO-0.5 samples and bulk HTc. However, at a first glance, a direct correlation 
between adsorbed $\mathrm{CO}_{2}$ and catalytic activity cannot be established (Figure 7c and Table 2).

Assuming that acetone self-condensation requires strong basic sites, an additional $\mathrm{CO}_{2}$ adsorption experiment was performed in order to determine their amount. For that purpose, a first $\mathrm{CO}_{2}$ adsorption was performed in static conditions at low pressures (as previously described) followed by an outgassing in high vacuum $\left(10^{-7}\right.$ Torr). Then, a second $\mathrm{CO}_{2}$ adsorption was performed. The difference between the first and second adsorptions gives the irreversibly chemisorbed $\mathrm{CO}_{2}$ on the strongest basic sites. Results are presented in Table 2.

They demonstrate the presence of strong basic sites in all the prepared HT/rGO nanocomposites, which were boosted respect to bulk HT in the HT/rGO-10 series of samples. A possible explanation can be given based on a greater number of lowcoordinated oxygens produced on the small MgAlOx crystals dispersed on the rGO matrix, both in intimate contact as seen by HRTEM. Particularly, HT/rGO-10CFD presented the smallest crystallite size and the highest amount of strong-strength basic sites. Accordingly, a correlation was found between strong basicity and activity in the self-condensation reaction, sample HT/rGO-10CFD having the highest conversion. This behavior is comparable to HT/rGO-0.5 series of catalysts as well, HT/rGO-0.5CFD showing the highest density of strong basic sites and catalytic activity.

The global rates of reaction (normalized per gram of HTc) of the HT/rGO-10 catalysts as a function of the number of strong basic sites are reported in Figure 7D and show a linear correlation. The reaction rate increases with the density of strong basic sites.

\section{Conclusions}

HT/rGO nanocomposites are likely to be applicable to a wide variety of applications, such as supercapacitors, adsorbents or electrocatalysts. Here it is presented, for the first time, a new application of $\mathrm{HT} / \mathrm{rGO}$ hybrid structures as active solid base catalysts. Both $\mathrm{CO}_{2}$ adsorption and test reaction in acetone self-condensation were performed to prove the basic character of these materials prepared by two different routes (i.e., direct coprecipitation and self-assembly) and dried either with temperature or freeze drying.

HRTEM analysis revealed the intimate contact between the hydrotalcite and graphene components in all the cases. However, it was found that coprecipitation led to more homogeneous HT/rGO nanocomposites, where HT nanoplatelets with markedly smaller size than those prepared by self-assembly, were well dispersed on the surface of the rGO nanosheets. On the other hand, samples dried in air at $80{ }^{\circ} \mathrm{C}$ developed more ordered structures by hydrogen bonding between both HT and rGO building blocks than freeze dried samples.

Besides the preparation and drying methods, an influence of the relative content of the GO and HT phases on the structural features of HT/rGO hybrids was also found. To evaluate the basic properties of the thermally activated HT/rGO hybrids both the 
adsorption of $\mathrm{CO}_{2}$ and the catalytic test reaction of acetone self-condensation were performed.

The different physico-chemical properties were mirrored in the catalytic behavior of the samples. After thermal activation HT/rGO-10 showed a two-fold enhanced catalytic activity than the bulk HTc. In particular, HT/rGO-10CFD which presented the lowest crystallite size and the highest amount of strong-strength basic sites led to the highest catalytic activity. Notably, the coprecipitated and freeze dried sample have the same behavior being the most active in the series with $\mathrm{HT} / \mathrm{rGO}=0.5: 1$ and 10:1 (HT/rGO0.5CFD and HT/rGO-10CFD).

The high activity in combination with the high tunability by modification of the preparation route makes the $\mathrm{HT} / \mathrm{rGO}$ nanocomposites promising candidates as base solid catalysts. The marked and tunable basic character of $\mathrm{HT} / \mathrm{rGO}$ materials demonstrated in this work opens a path to new applications of graphene-based nanocomposites, which can widen with the use of multifunctional $\mathrm{HT} / \mathrm{rGO}$ nanocomposites.

\section{Acknowledgements}

This research has been supported by the People Programme (Marie Curie Actions) of the European Union's Seventh Framework Programme (FP7/2007-2013) and from ACCIÓ under REA (grant agreement $n^{\circ}$ 600388) through the grant TECSPR13-1-0038, Tecniospring-Marie Curie fellowship Programme. JL is Serra Hunter Fellow and is grateful to ICREA Academia program and MINECO (grant ENE2015-63969-R).

\footnotetext{
${ }^{1}$ Rives, V., 2001.Layered Double Hydroxides: Present and Future. In: Rives, V. (Ed.), Nova Science Publishers Co., Inc., New York

${ }^{2}$ Cavani, F., Trifirò, F., Vaccari, A., 1991. Catal. Today 11, 173-301.

${ }^{3}$ Chimentão, R.J., Abelló, S., Medina, F., Llorca, J., Sueiras, J.E., Cesteros, Y., Journal of Catalysis 252, 249-257

${ }^{4}$ Álvarez, M.G., Segarra, A.M., Contreras, S., Sueiras, J.E., Medina, F., Figueras, F., Chemical Engineering Journal 161, 340-345

${ }^{5}$ Li, L., Shi, J., 2008. Chem. Commun. 996-998.

${ }^{6}$ Dubey, A., 2007. Green Chem. 9, 424-426.

7 Álvarez, M.G., Plíšková, M., Segarra, A.M., Medina, F., Figueras, F., 2012. Appl. Catal. B Environ. 113-114, 212-220.

${ }^{8}$ M.R. Othman, N.M. Rasid, W.J.N. Fernando, Chem. Eng. Sci., 61 (2006) 1555

${ }^{9}$ Z. Lü, F. Zhang, X. Lei, L. Yang, S. Xu, X. Duan, Chem. Eng. Sci., 63 (2008) 4055

${ }^{10}$ L.B. Modesto-López, R.J. Chimentão, M.G. Álvarez, J. Rosell-Llompart, F. Medina, J.Llorca. Appl. Clay Sci., 101 (2014) 461

${ }^{11}$ Winter, F., van Dillen, A.J., de Jong, K.P., 2005 Chem. Commun. 3977-3979.

${ }^{12}$ M.G. Álvarez, A.M. Frey, J.H. Bitter, A.M. Segarra, K.P. de Jong, F. Medina, Appl. Catal. B: Environ. 134- 135 (2013) 231-237

${ }^{13}$ a)A. Garcia-Gallastegui, D. Iruretagoyena, V. Gouvea, M. Mokhtar, A.M. Asiri, S.N. Basahel, S.A. AlThabaiti, A.O. Alyoubi, D. Chadwick, M.S.P. Shaffer. Chem. Mater. 2012, 24, 4531-4539; b) A. GarciaGallastegui, D. Iruretagoyena, M. Mokhtar, A.M Asiri, S.N. Basahel, S.A. Al-Thabaiti, A.O. lyoubi, D. Chadwick, M.S.P. Shaffer, J. Mater. Chem. 2012, 22 (28), 13932-13940.

${ }^{14}$ Hummers, JACS (1958) 1339.

${ }^{15}$ M.G. Álvarez, R.J. Chimentão, F. Figueras, F. Medina. Applied Clay Science 58 (2012) 16-24.
} 
${ }^{16}$ R. Ma, X. Liu, J. Liang, Y. Bamdo, T. Sasaki. Adv. Mater., 26 (2014) 4173.

${ }^{17}$ W. Ma, R. Ma, C. Wang, J. Liang, X. Liu, K. Zhou, T. Sasaki. ACS Nano, 9 (2015) 1997.

${ }^{18}$ K. Bhowmik, S. Pramanik, S.K Medda, G. De. Mater.Chem., 22 (2012) 24690.

${ }^{19}$ M. Acik, G. Lee, C. Mattevi, M. Chhowalla, K. Cho, Y. J. Chabal. Nat. Mater., 9 (2010) 840.

${ }^{20}$ T. Kloprogge R.L. Frost.J. Solid State Chem., 146 (1999) 506.

${ }^{21}$ A.C. Ferrari.Solid State Commun.143 (2007) 47.

${ }^{22}$ M. A. Pimenta, G. Dresselhaus, M. S. Dresselhaus, L. G. Cançado, A. Jorio, R. Saito. Phys. Chem. Chem. Phys., 9 (2007) 1276

${ }^{23}$ Q. Fang, B. Chen. J. Mater. Chem A, 2 (2014) 8941.

${ }^{24}$ Y. Xu, Q. Wu, Y. Sun, H. Bai, G. Shi, ACS Nano, 4 (2010) 7358.

${ }^{25}$ H-P.Cong, X-C.Ren, P. Wang, S-H.Yu, ACS Nano, 6 (2012) 2693.

${ }^{26}$ a) G.G. Podrebarac, F.T.T. Ng, G.L. Rempel, Chem. Eng. Sci. 52 (1997) 2991; b) F. Prinetto, D. Tichit, R.Teissier, B. Coq, Catal. Today 55 (2000) 103; c) F. Winter, V. Koot, A.J. van Dillen, J.W. Geus, K.P. de Jong, J. Catal. 236 (2005) 91.

${ }^{27}$ P. Kustrowski, D. Sułkowska, L.Chmielarz,A.Rafalska-Łasocha,B.Dudek,R.Dziembaj, Micropor. Mesopor.Mater.78 (2005) 11.

${ }^{28}$ F. Prinetto, G. Ghiotti, R. Durand, D.Tichit, J. Phys. Chem. B 104(2000) 11117.

${ }^{29}$ P. Kustrowski, D. Sułkowska, R. Pytlowany, R.Dziembaj, React.Kinet.Catal.Lett. 81 (2004) 3.

${ }^{30}$ Diana Iruretagoyena, Milo S. P. Shaffer, David Chadwick Adsorption (2014) 20:321-330 

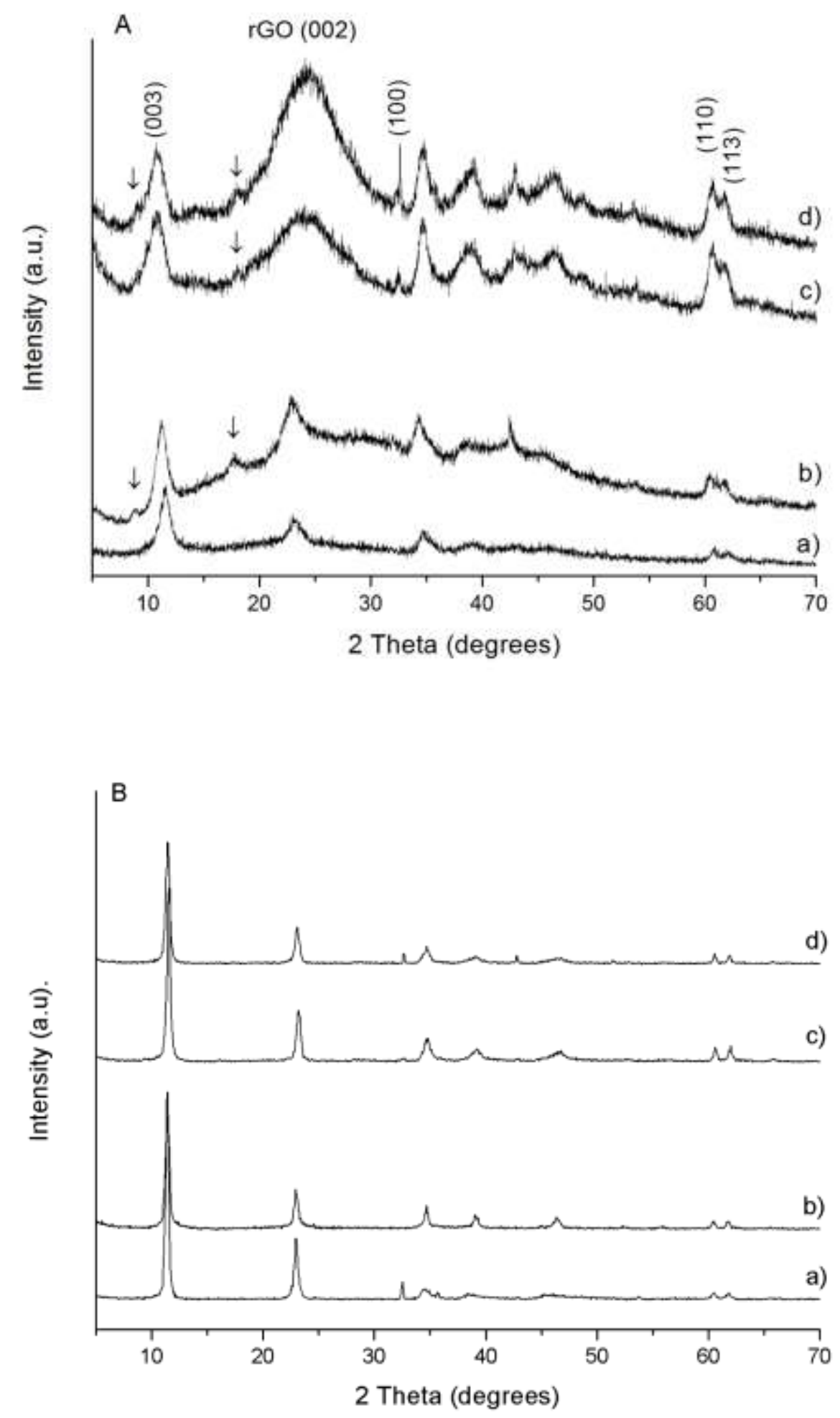

Figure 1. A) XRD patterns of $\mathrm{HT} / \mathrm{rGO}$ hybrids with $\mathrm{rGO}$ : $\mathrm{HT}=1: 0.5$ a) $\mathrm{HT} / \mathrm{rGO}-0.5 \mathrm{C} 80$; b) HT/rGO-0.5CFD; c) HT/rGO-0.5SA80 and d) HT/rGO-0.5SAFD. B) XRD patterns of HT/rGO hybrids with $\mathrm{rGO}: \mathrm{HT}=1: 10$ a) $\mathrm{HT} / \mathrm{rGO}-10 \mathrm{C} 80$; b) HT/rGO-10CFD; c) HT/rGO-10SA80 and d) HT/rGO-10SAFD. 

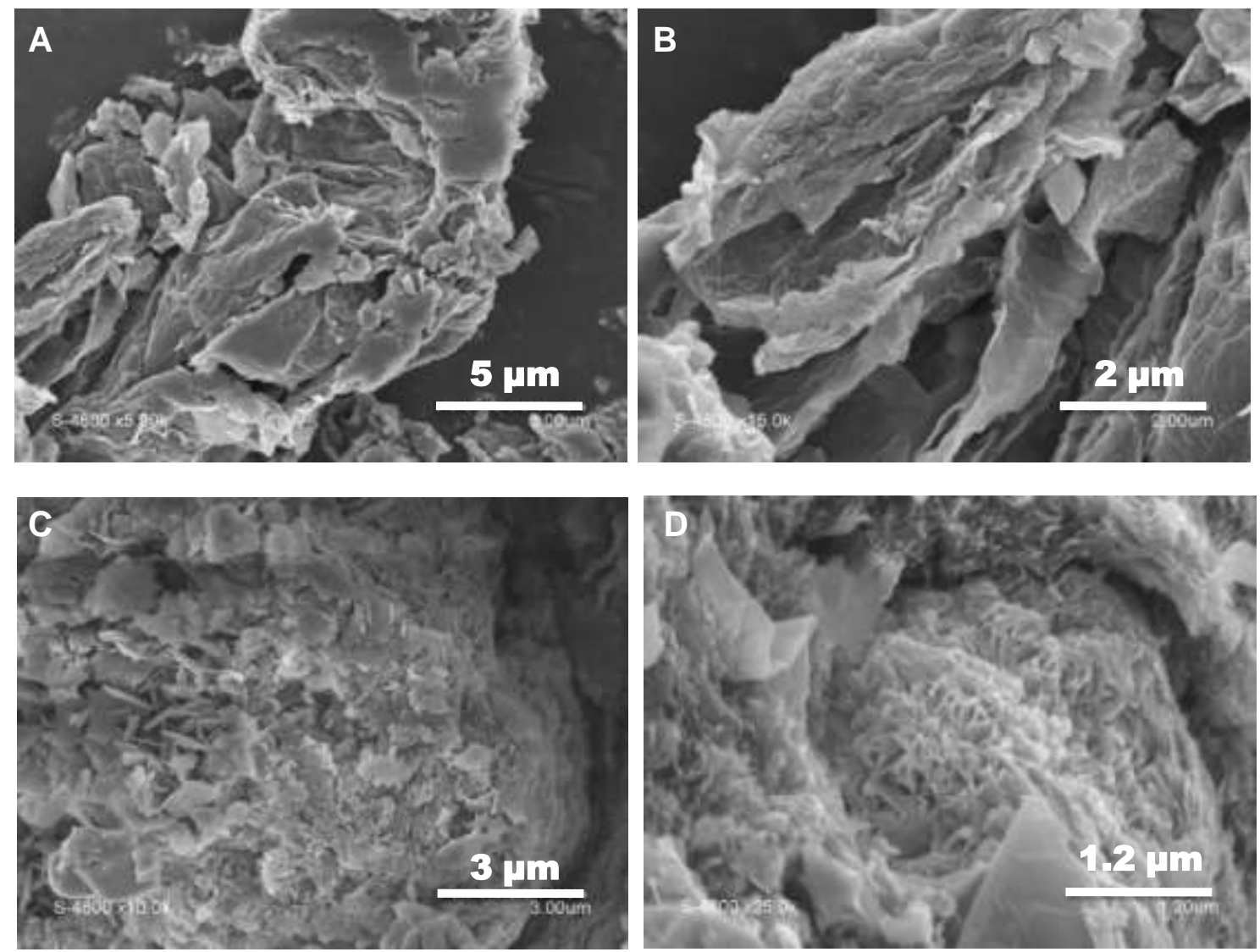

Figure 2. SEM micrographies of (A and B) HT/rGO-0.5C80 and (C and D) HT/rGO$0.5 \mathrm{SA} 80$ dried at $80^{\circ} \mathrm{C}$.

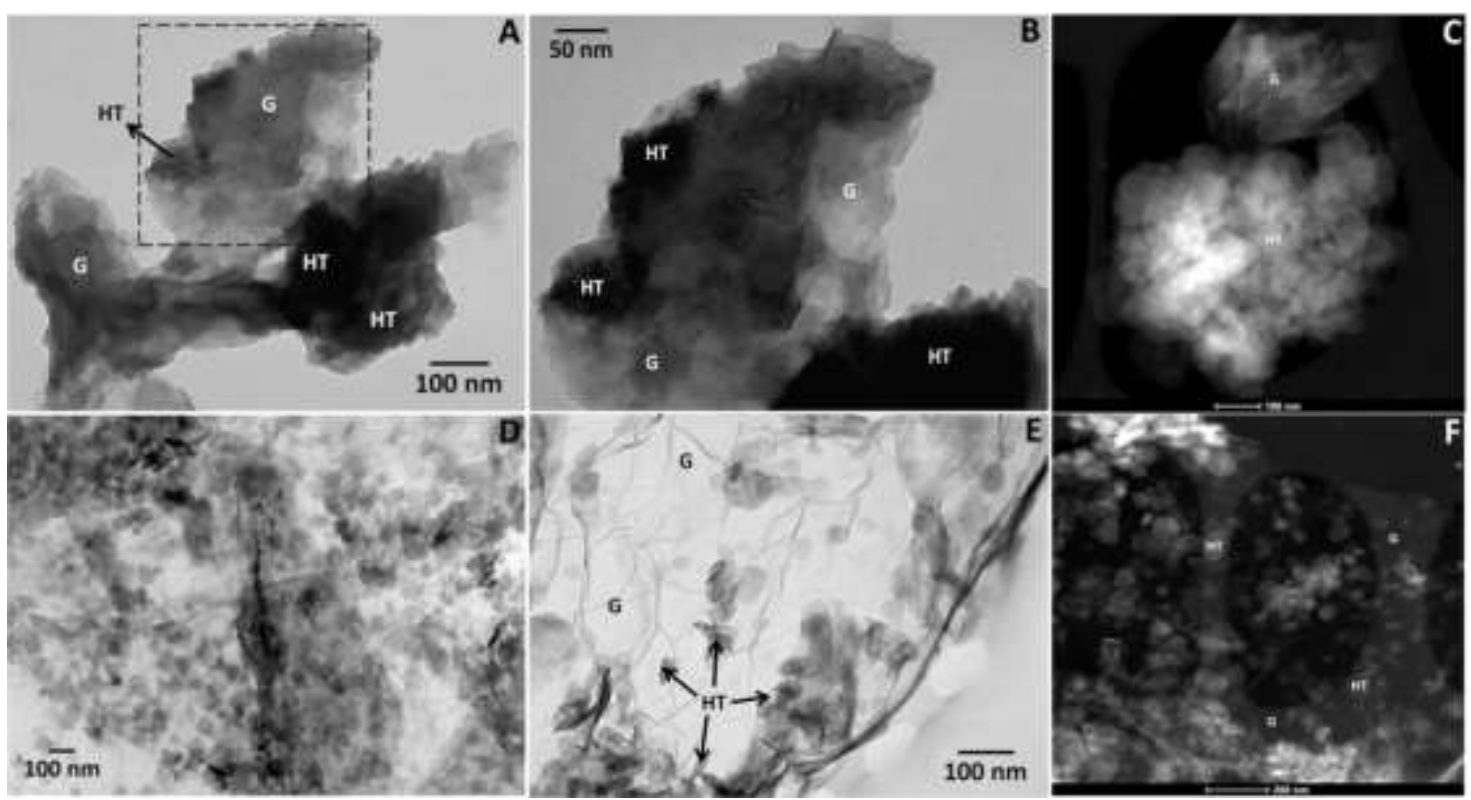

Figure 3. A, B) TEM and C) STEM-HAADF micrographies of sample HT/rGO-0.5C80 (dried at $80^{\circ} \mathrm{C}$ ); D, E) TEM and F) STEM-HAADF micrographies of sample HT/rGO$0.5 \mathrm{CFD}$ (freeze dried). 

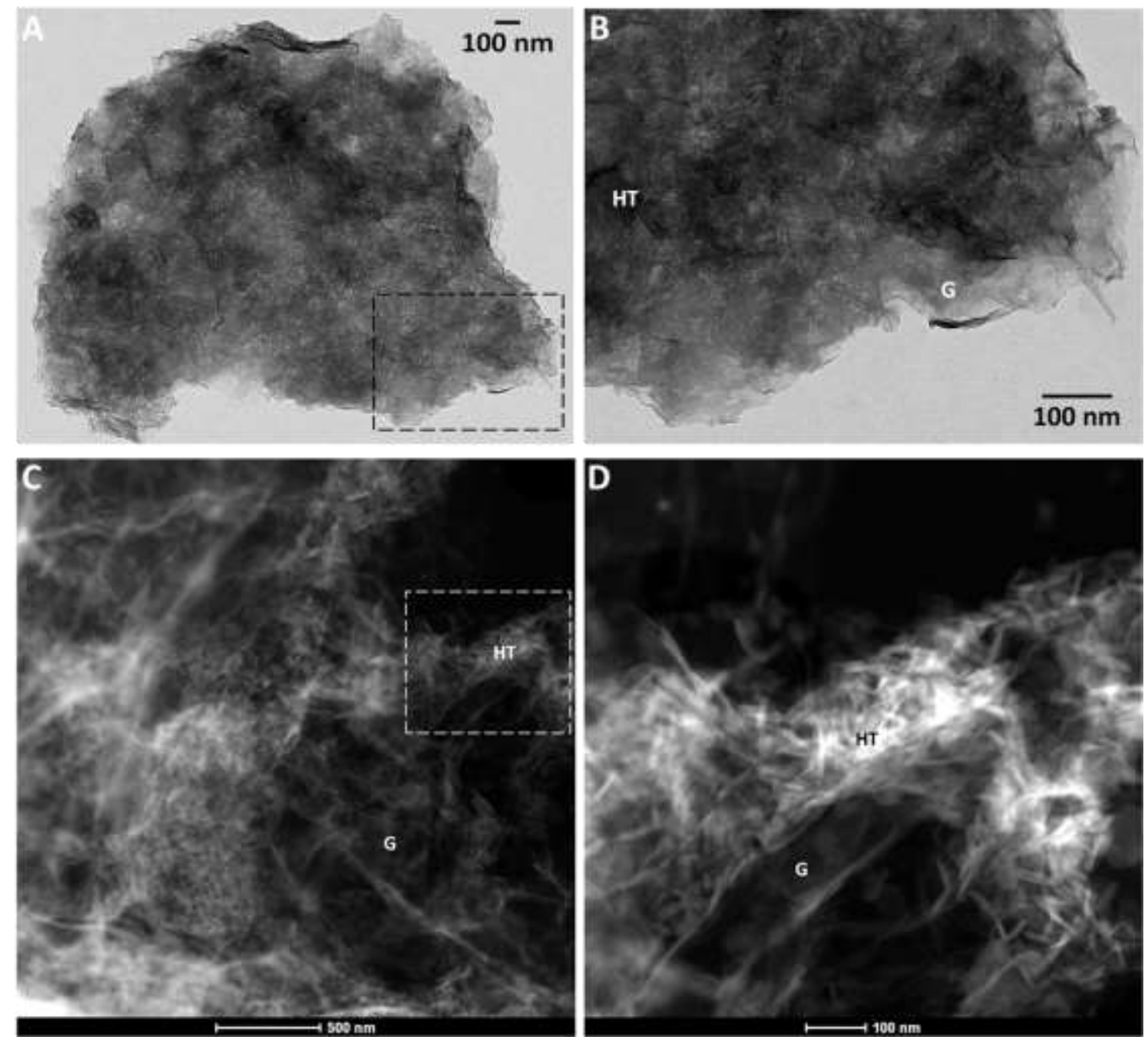

Figure 4. A, B) TEM and C, D) STEM-HAADF micrographies of the hybrid material $\mathrm{HT} / \mathrm{rGO}-0.5 \mathrm{SA} 80$ (prepared by self-assembly and dried at $80{ }^{\circ} \mathrm{C}$ ). 


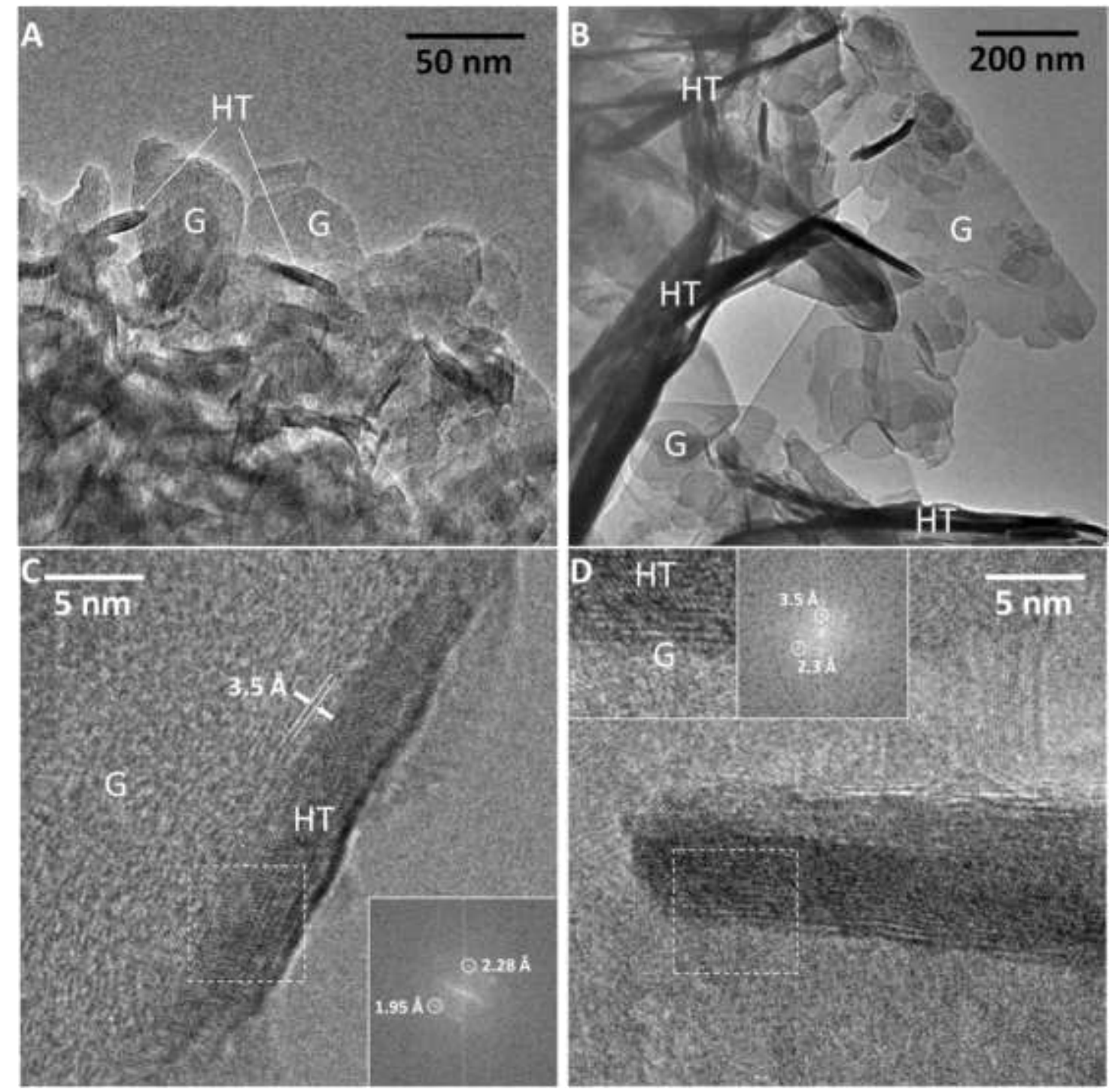

Figure 5. HRTEM images of HT/rGO-10 samples prepared by different routes: A, C) HT/rGO-10CFD (coprecipitation and freeze drying) and B, D) HT/rGO-10SAFD (selfassembly and freeze drying). 

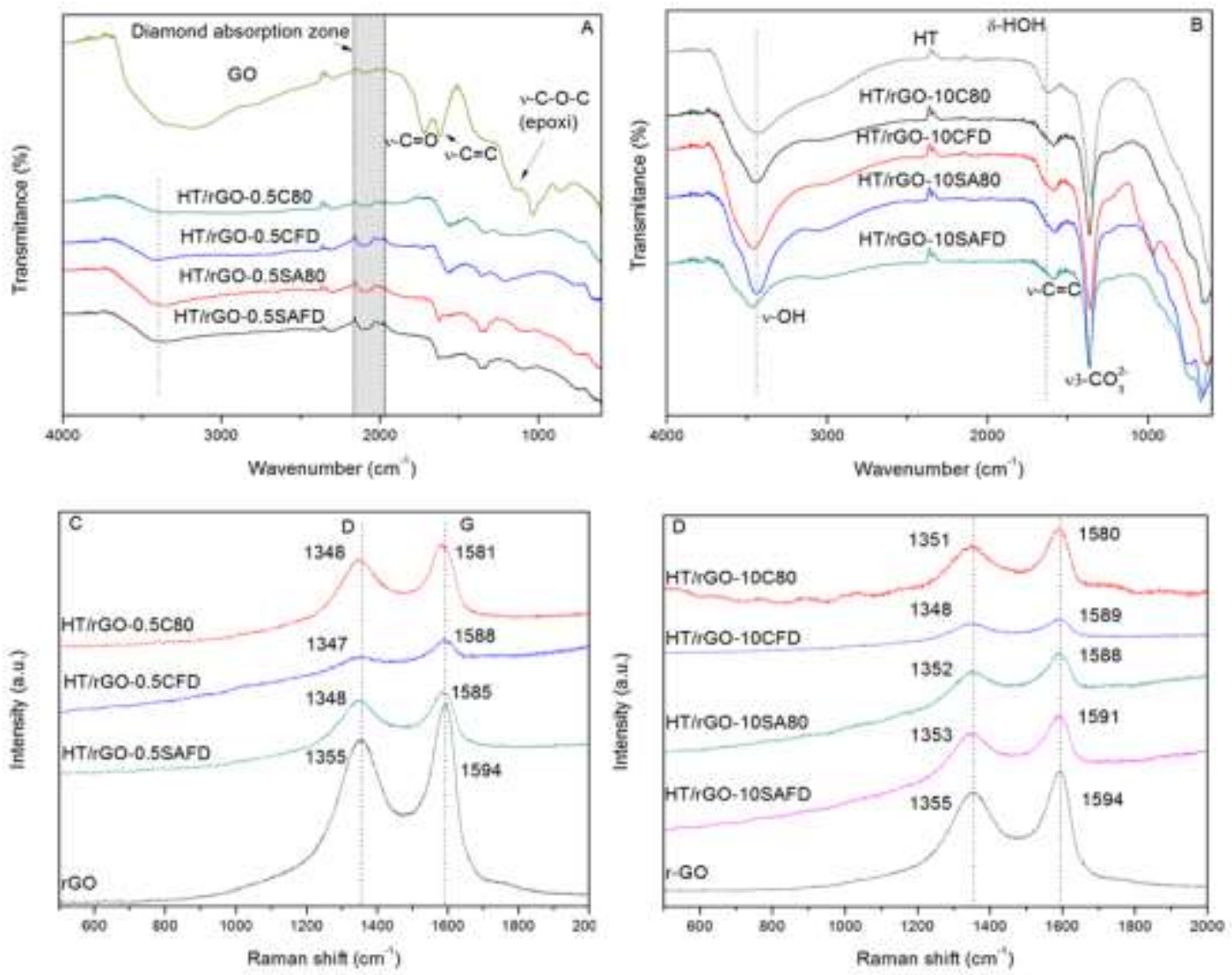

Figure 6. A and B) ATR-FTIR spectra and C and D) Raman spectra of HT/rGO samples prepared and dried by the different routes and with different HT:rGO ratios. 

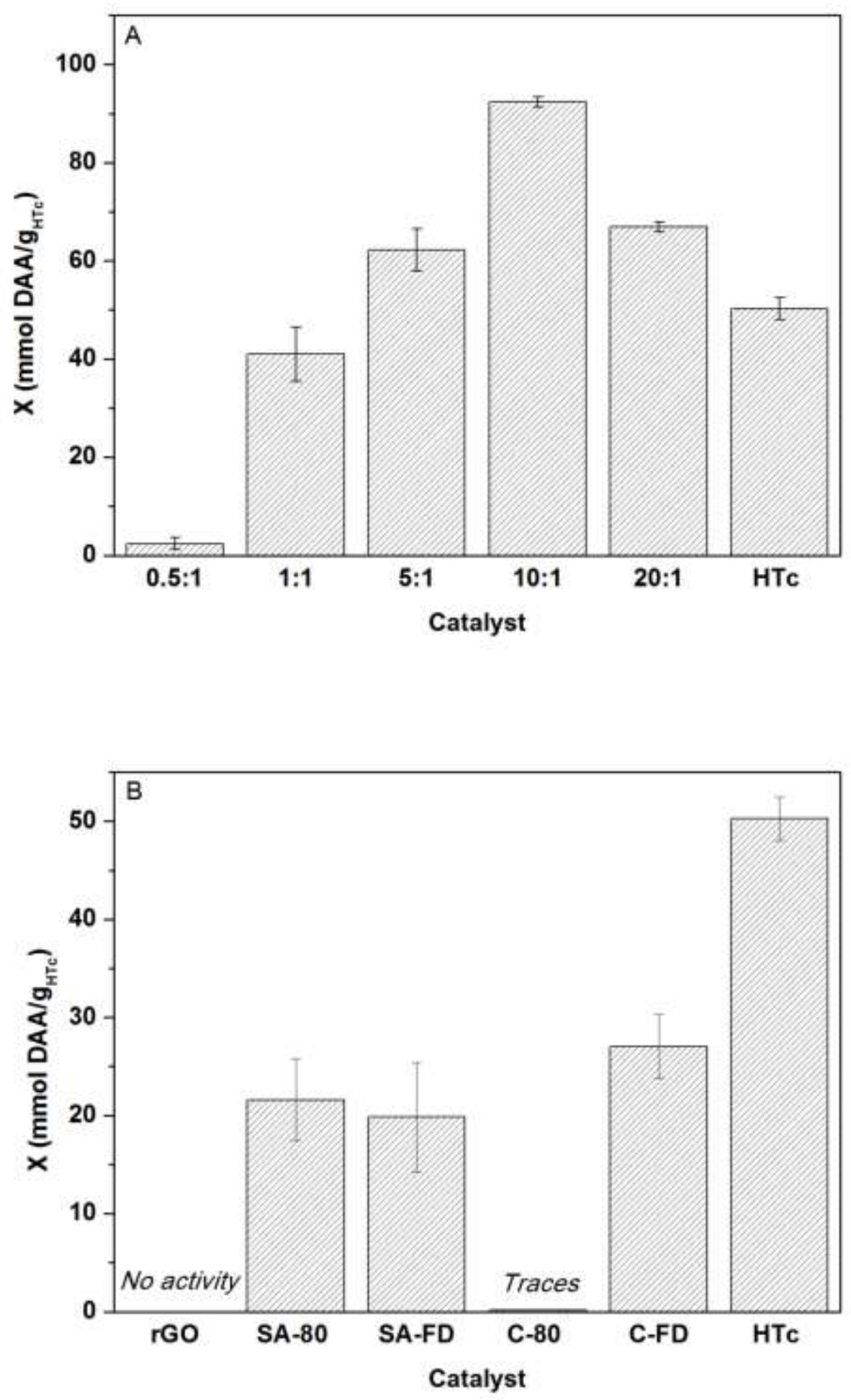

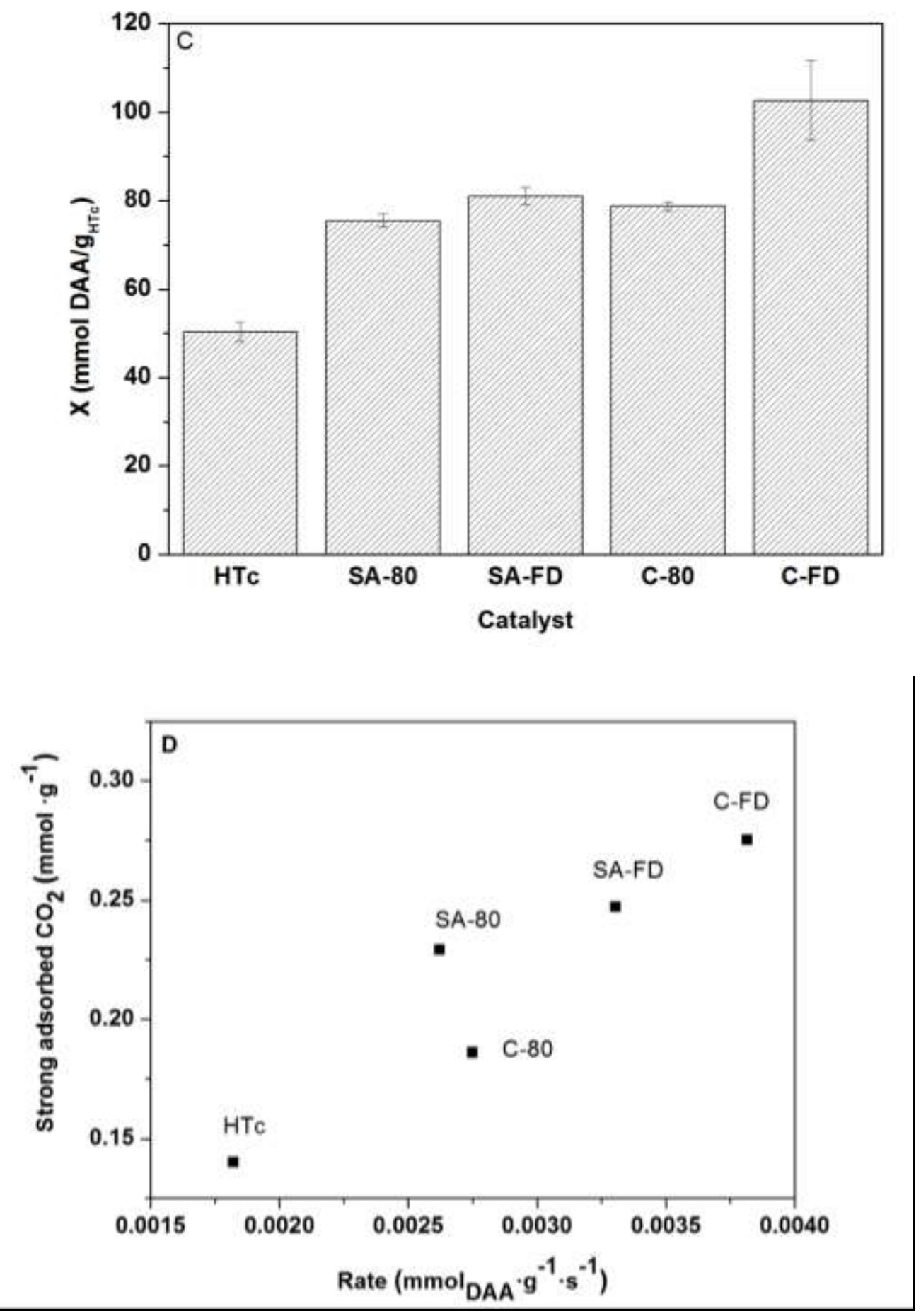

Figure 7. Catalytic behavior of the $\mathrm{HT} / \mathrm{rGO}$ hybrid materials in the self-condensation of acetone. A) HT/rGO-xC80 hybrid catalysts with $\mathrm{HT}: \mathrm{rGO}=0.5: 1,1: 1,5: 1,10: 1$ and 20:1; B) HT/rGO-0.5 hybrid catalysts prepared by different methods; C) HT/rGO-10 hybrid catalysts prepared by different methods; D) Influence of the number of strongstrength basic sites on the activity of HT/rGO-10 catalysts. 